GEF-Th-24/2006

\title{
The QCD Parton Model: a Useful Approximation
}

\author{
Elvio Di Salvo \\ Dipartimento di Fisica and I.N.F.N. - Sez. Genova, Via Dodecaneso, 33 \\ - 16146 Genova, Italy
}

\begin{abstract}
Approximate relations among transverse momentum dependent quark distribution functions are established in the framework of the QCD parton model. The validity of such results survives QCD evolution effects, owing to the Politzer theorem on equations of motion. Furthermore the model fixes an energy scale, involved in the parametrization of the correlator, which determines the $Q^{2}$ dependence of the azimuthal asymmetries in inclusive reactions. Some of the present data - the $\cos 2 \phi$ asymmetry in unpolarized Drell-Yan, the $\sin 2 \phi$ single spin asymmetry in semi-inclusive deep inelastic scattering (SIDIS) and the $\cos \phi$ asymmetry in unpolarized SIDIS - support model predictions. Further measurements of SIDIS and Drell-Yan asymmetries are suggested, in particular the double spin asymmetry in semi-inclusive deep inelastic scattering, which allows to determine approximately the proton transversity.
\end{abstract}

PACS numbers: 13.85.Qk, 13.88.+e 


\section{Introduction}

The quark-quark correlator $[1,2]$ is a fundamental tool for calculating cross sections in inclusive high energy reactions. It may be parametrized according to the Dirac algebra, using the available vectors. We distinguish between the common correlator, which depends only on the longitudinal fractional momentum, and the transverse momentum dependent one. In the former case the correlator consists of 9 components[3],

while in the latter case the number of independent functions is $32[4,5]$ - two for any Dirac operator - of which 23 are washed out upon integration over transverse momentum. These last functions contain the dimensionless four-vector

$$
\eta_{\perp}=p_{\perp} / \mu_{0}
$$

where $p_{\perp}$ is the transverse four-momentum of the active quark with respect to the hadron and $\mu_{0}$ an undetermined energy scale, introduced for dimensional reasons. We shall fix this parameter by comparison with the limiting case of the QCD parton model, which provides also useful approximate relations between different soft functions. Some predictions of this model are tested against data and further measurements are suggested.

First of all we introduce the quark-quark correlator, $\Phi$, discussing its normalization. Secondly, we parametrize $\Phi$ according to the Dirac components. Then we write the density matrix of a quark in the approximation of the QCD parton model. Comparison with the general parametrization of $\Phi$ allows to determine $\mu_{0}$ and implies approximate relations among transverse momentum distributions. Lastly we compare our results with present data and suggest for them further experimental tests.

\section{Parametrization of the correlator}

The correlator is involved in a typical calculation of inclusive cross section - for example, deep inelastic scattering (DIS), semi-inclusive DIS (SIDIS), or Drell-Yan (DY) i. e.,

$$
\frac{d \sigma}{d \Gamma}=\frac{(4 \pi \alpha)^{2}}{4 \mathcal{F} Q^{4}} L^{\mu \nu} W_{\mu \nu}
$$


Here $d \Gamma$ is the phase space element, $\alpha$ the fine structure constant, $Q^{2}$ the virtuality of the exchanged photon and $\mathcal{F}$ the flux factor. Moreover $L^{\mu \nu}$ and $W^{\mu \nu}$ are respectively the leptonic and hadronic tensor. In particular, the hadronic tensor reads, at zero order in QCD,

$$
W^{\mu \nu}=c \sum_{a} e_{a}^{2} \int d^{2} p_{\perp} \operatorname{Tr}\left[\Phi_{A}^{a}\left(x_{a}, \mathbf{p}_{\perp}\right) \gamma^{\mu} \Phi_{B}^{b}\left(x_{b}, \mathbf{q}_{\perp}-\mathbf{p}_{\perp}\right) \gamma^{\nu}\right]
$$

Here $c=1$ and the flavor $b$ coincides with $a$ for DIS (and SIDIS), while $c=1 / 3$ and $b=\bar{a}$ for DY. Each correlator is normalized so as to reduce to the usual spin density matrix in absence of parton-parton interactions. For a nucleon we have

$$
\Phi=\Phi_{e}+\Phi_{o}
$$

where $\Phi_{e}$ is even under time reversal and $\Phi_{o}$ is odd under the same transformation. $\Phi_{e}$ reads, up to and including twist $3[2,6,5]$,

$$
\Phi_{e} \simeq \Phi_{e}^{(2)}+\Phi_{e}^{(3)}
$$

with

$$
\begin{aligned}
\Phi_{e}^{(2)} & =\frac{\mathcal{P}}{\sqrt{2}}\left\{f_{1} \not h_{+}+\left(\lambda g_{1 L}+\lambda_{\perp} g_{1 T}\right) \gamma_{5} h_{+}+\frac{1}{2} h_{1 T} \gamma_{5}\left[\$_{\perp}, \not h_{+}\right]\right. \\
& \left.+\frac{1}{2}\left(\lambda h_{1 L}^{\perp}+\lambda_{\perp} h_{1 T}^{\perp}\right) \gamma_{5}\left[h_{\perp}, \not h_{+}\right]\right\}
\end{aligned}
$$

and

$$
\begin{aligned}
\Phi_{e}^{(3)} & =\frac{1}{2}\left(f^{\perp}+\lambda g_{L}^{\perp} \gamma_{5}+\lambda_{\perp} g_{T}^{\perp} \gamma_{5}\right) p_{\perp}+\frac{1}{4} \lambda_{\perp} h_{T}^{\perp} \gamma_{5}\left[\$_{\perp}, \not p_{\perp}\right] \\
& +\frac{1}{2} x M\left\{e+g_{T}^{\prime} \gamma_{5} \$_{\perp}+\frac{1}{2}\left(\lambda h_{L}+\lambda_{\perp} h_{T}\right) \gamma_{5}\left[h_{-}, \not h_{+}\right]\right\}
\end{aligned}
$$

On the other hand, the twist- 2 component of $\Phi_{o}$ amounts to[7, 8]

$$
\Phi_{o} \simeq \frac{\mathcal{P}}{\sqrt{2}}\left\{f_{1 T}^{\perp} \epsilon_{\mu \nu \rho \sigma} \gamma^{\mu} n_{+}^{\nu} \eta_{\perp}^{\rho} S_{\perp}^{\sigma}+i h_{1}^{\perp} \frac{1}{2}\left[h_{\perp}, h_{+}\right]\right\} .
$$

In formulae (6) to (8) we have used the notations of refs. [2, 9] for the "soft" functions, but with different normalizations, as we shall specify below. They are functions of $x$ and of $p_{\perp}^{2}$, where $x=p^{+} / P^{+}$and $p_{\perp}$ are, respectively, the longitudinal fractional 
momentum and the transverse four-momentum of the quark. We have chosen a frame where, in light cone coordinates, the four-momentum of the nucleon is $P \equiv$ $\left(P^{+}, P^{-}, \mathbf{0}\right)$, the quark four-momentum is $p \equiv\left(p^{+}, p^{-}, \mathbf{p}_{\perp}\right)$ and $p_{\perp} \equiv\left(0,0, \mathbf{p}_{\perp}\right) . n_{ \pm}$ are lightlike vectors, such that $n_{+} \cdot n_{-}=1$, whose space components are directed along (+) or opposite to (-) the nucleon momentum. Moreover $P^{2}=M^{2}$ and

$$
S=\lambda \frac{P}{M}+S_{\perp}
$$

is the Pauli-Lubanski (PL) vector of the nucleon; one has $S^{2}=-1, \lambda=-S \cdot n_{0}$ and $n_{0} \equiv 1 / \sqrt{2}(1,-1,0)$ in the nucleon rest frame. Thirdly,

$$
\mathcal{P}=\frac{1}{\sqrt{2}} p \cdot n_{-}, \quad \lambda_{\perp}=-S \cdot \eta_{\perp},
$$

$\eta_{\perp}$ being given by eq. 1. Lastly, the energy scale $\mu_{0}$, encoded in $\eta_{\perp}$, has been introduced in such a way that all functions involved in the parametrization of $\Phi$ have the dimensions of a probability density. This scale - defined for the first time in ref.[10], where it was denoted by $m_{D}$ - determines the normalization of the functions which depend on $\eta_{\perp}$; therefore $\mu_{0}$ has to be chosen in such a way that these functions may be interpreted just as probability densities. We can reasonably assume this parameter to be independent of the perturbative interactions among partons.

We conclude this section with a comparison between the normalization of our functions and the one of Mulders et al.. We distinguish between the functions which are not multiplied by $\eta_{\perp}$ and those which are multiplied by that vector. As regards the former category, we have, e. g.,

$$
f_{1}=f_{1}^{m} / x, \quad g_{1 L}=g_{1 L}^{m} / x, \quad h_{1 T}=h_{1 T}^{m} / x,
$$

where the superscript $m$ refers to the Mulders convention. Concerning the second group of functions, the relations are of the type, for example,

$$
g_{1 T}=g_{1 T}^{m} M /\left(\mu_{0} x\right), \quad h_{1 L}^{\perp}=h_{1 L}^{\perp m} M /\left(\mu_{0} x\right) .
$$




\section{Density matrix in QCD parton model}

Now we write the density matrix of a confined quark, but free of interactions with other partons. To this end, we inspire to the one of a free spin- $1 / 2$ particle, i. e.,

$$
\rho_{f}=\frac{1}{2}(\not p+m)\left(1+\gamma_{5} \$^{\prime}\right),
$$

where $p, m$ and $S^{\prime}$ are respectively the 4-momentum, the mass and the Pauli-Lubanski 4 -vector of the fermion. We rewrite eq. (13) as

$$
\rho_{f}=\frac{1}{2}(\not p+m)\left(1+\gamma_{5} \phi_{\|}^{\prime}+\gamma_{5} \phi_{\perp}^{\prime}\right)
$$

where $S_{\|}^{\prime}=\lambda^{\prime} p / m, S_{\perp}^{\prime}=S^{\prime}-S_{\|}^{\prime}, \lambda^{\prime}=\hat{\mathbf{p}} \cdot \hat{\mathbf{s}}$ and $\hat{\mathbf{p}}$ and $\hat{\mathbf{s}}$ are unit vectors in the directions, respectively, of the quark momentum and of the spin in the particle rest system. In order to generalize eq. (14) to the case of interest, we take into account three elements:

a) the internal structure of the nucleon is sensitive both to longitudinal and to transverse polarization, in an independent way;

b) the spin of a massive particle has to be defined in its rest frame, therefore the PL vector of the quark does not coincide with the one of the nucleon[11];

c) the quark can be treated as if it were on shell, owing to the equations of motion; we shall discuss this point below.

Then

$$
\rho_{c}=\frac{1}{2}\left(\not p+m_{q}\right)\left[q\left(x, p_{\perp}^{2}\right)+\Delta q\left(x, p_{\perp}^{2}\right) \gamma_{5} \phi_{\|}^{q}+\Delta_{T} q\left(x, p_{\perp}^{2}\right) \gamma_{5} \$_{\perp}^{q}\right] .
$$

Here $S_{\|}^{q}$ and $S_{\perp}^{q}$ are defined in such a way that, in the quark rest frame, they coincide respectively with $S_{\|}=S-S_{\perp}$ and $S_{\perp}$ in the nucleon rest frame. Therefore

$$
S_{\|}^{q}=\lambda\left(\frac{p}{m_{q}}-\bar{\eta}_{\perp}\right)+O\left(\bar{\eta}_{\perp}^{2}\right), \quad S_{\perp}^{q}=S_{\perp}+\bar{\lambda}_{\perp} \frac{p}{m_{q}}+O\left(\bar{\eta}_{\perp}^{2}\right),
$$

with $\bar{\eta}_{\perp}=p_{\perp} / \mathcal{P}$ and $\bar{\lambda}_{\perp}=-S \cdot \bar{\eta}_{\perp}$. Moreover, we have set

$$
\begin{aligned}
q\left(x, p_{\perp}^{2}\right) & =q_{+}\left(x, p_{\perp}^{2}\right)+q_{-}\left(x, p_{\perp}^{2}\right)=q_{\uparrow}\left(x, p_{\perp}^{2}\right)+q_{\downarrow}\left(x, p_{\perp}^{2}\right), \\
\Delta q\left(x, p_{\perp}^{2}\right) & =q_{+}\left(x, p_{\perp}^{2}\right)-q_{-}\left(x, p_{\perp}^{2}\right), \\
\Delta_{T} q\left(x, p_{\perp}\right) & =q_{\uparrow}\left(x, p_{\perp}^{2}\right)-q_{\downarrow}\left(x, p_{\perp}^{2}\right) .
\end{aligned}
$$


Here $q_{ \pm}\left(x, p_{\perp}^{2}\right)$ and $q_{\uparrow(\downarrow)}\left(x, p_{\perp}^{2}\right)$ are the probability densities of finding a quark with, respectively, a positive $(+)$ or negative $(-)$ helicity and a positive $(\uparrow)$ or negative $(\downarrow)$ transversity, the sign of the latter being determined by the sign of the scalar product between the quark transversity and the nucleon transversity. Eq. (15) can be obtained as a limiting expression[12] of the correlator in a gauge theory, $i$. e.,

$$
\Phi=\int \Phi^{\prime}(p ; P, S) d p^{-}
$$

where the matrix elements of $\Phi^{\prime}(p ; P, S)$ are defined as

$$
\Phi_{i j}^{\prime}(p ; P, S)=\int \frac{d^{4} x}{(2 \pi)^{4}} e^{i p x}\left\langle P, S\left|\bar{\psi}_{j}(0) \mathcal{L}(x) \psi_{i}(x)\right| P, S\right\rangle .
$$

Here $\psi$ is the quark field and $|P, S\rangle$ is a state of the nucleon. Moreover

$$
\mathcal{L}(x)=\operatorname{Pexp}\left[-i g \Lambda_{\mathcal{P}}(x)\right], \quad \text { with } \quad \Lambda_{\mathcal{P}}(x)=\int_{0}^{x} \lambda_{a} A_{\mu}^{a}(z) d z^{\mu},
$$

is the gauge link operator. Here "P" denotes the path-ordered product along the integration contour $\mathcal{P}, \lambda_{a}$ and $A_{\mu}^{a}$ being respectively the Gell-Mann matrices and the gluon fields. The link operator depends on the choice of $\mathcal{P}$, which has to be fixed so as to make a physical sense. However, for our aims we can neglect the details of the contour. Indeed, in the limit for $g \rightarrow 0$, that is, for noninteracting quarks, $\mathcal{L}(x)$ tends to 1 . Moreover, in that limit, the quark may be treated as an on-shell particle, as shown also by Qiu[13] via equations of motion[14]; see also ref.[15]. It can be shown[12] that in this limit $\Phi$ tends to $\rho_{c}$. Another important consequence of the

equations of motion is that they survive renormalization, therefore eq. (15) is a good approximation to the correlator even if QCD evolution of the distribution functions is taken into account.

\section{Approximate equalities among "soft" functions}

Now we compare the density matrix (15) with the general parametrization (6) of the twist-2, T-even component of $\Phi$. To this end we consider projections of both matrices over the various Dirac components, $i$. e., for a given Dirac operator $\Gamma$,

$$
\Phi^{\Gamma}=\frac{1}{2} \operatorname{Tr} \Gamma \Phi .
$$


First of all, $\Gamma=\gamma^{+}, \gamma_{5} \gamma^{+}$and $\gamma_{5} \gamma^{+} \gamma_{i}(i=1,2)$ yield

$$
f_{1}=q, \quad g_{1 L}=\Delta q, \quad h_{1 T}=\Delta_{T} q
$$

and

$$
h_{1 L}^{\perp} \approx-\frac{\mu_{0}}{\mathcal{P}} \Delta q, \quad g_{1 T} \approx h_{1 T}^{\perp} \approx \frac{\mu_{0}}{\mathcal{P}} \Delta_{T} q .
$$

The last equalities hold approximately in the limit of $m_{q}=0$. In order to determine $\mu_{0}$, we observe that the functions $g_{1 T}, h_{1 L}^{\perp}$ and $h_{1 T}^{\perp}$, involved in formulae (25), are twist 2 , therefore they may be interpreted as quark densities, as well as $\Delta q$ and $\Delta_{T} q$. For example, $g_{1 T}$ is the helicity density of a quark in a tranversely polarized nucleon. Therefore it is natural to fix $\mu_{0}$ in such a way that such functions are normalized like $\Delta q$ or $\Delta_{T} q$. This implies, neglecting the quark mass,

$$
\mu_{0}=\mathcal{P}=\frac{1}{\sqrt{2}} p \cdot n_{-}
$$

This result differs from the treatments of previous authors $[2,16]$, who assume $\mu_{0}=M$. Moreover, it is interesting to consider also the projections over twist-3 operators, in particular $\Gamma=\gamma_{i}(i=1,2)$. This yields

$$
f^{\perp} \approx f_{1}=q
$$

which is known as the Cahn effect $[17,18]$. We have neglected quark-gluon interactions[2], which could modify, in principle, equalities (24), (25) and (27), in particular the last one, concerning a twist 3 operator. However, as we shall see, comparison with data, wherever possible, suggests that such equalities are approximately verified. On the contrary, the projection over $\Gamma=\gamma_{5} \gamma_{i}(i=1,2)$ yields (after integration over $\mathbf{p}_{\perp}$ )

$$
g_{T}(x)=\frac{m_{q}}{x M} h_{1}(x)
$$

In this case the contribution of the QCD parton model is very small: $m_{q}$ is negligible for $u$ - and $d$-quarks, while for $s$-quarks $h_{1}$ is predicted to be small, because sea quarks are produced mainly by annihilation of gluons, whose transversity is zero in a nucleon. Therefore the contribution of quark-gluon interactions, neglected in our model, becomes prevalent in this case, as well as for $\Gamma=1$ and $\gamma_{5} \gamma_{+} \gamma_{-}$, corresponding 
respectively to $e$ and $h_{L}$. Such interactions will be discussed below. Eq. (28) - similar to some expressions given in the literature[19, 2] - establishes a relation between transversity and transverse spin. Indeed, the two quantities are related to each other, as can be seen also from eq. (14) for a free fermion. But, unlike transversity, the transverse spin operator is chiral even and does not commute with the free hamiltonian of a quark[3]: in QCD parton model it is proportional to the quark rest mass, which causes chirality flip.

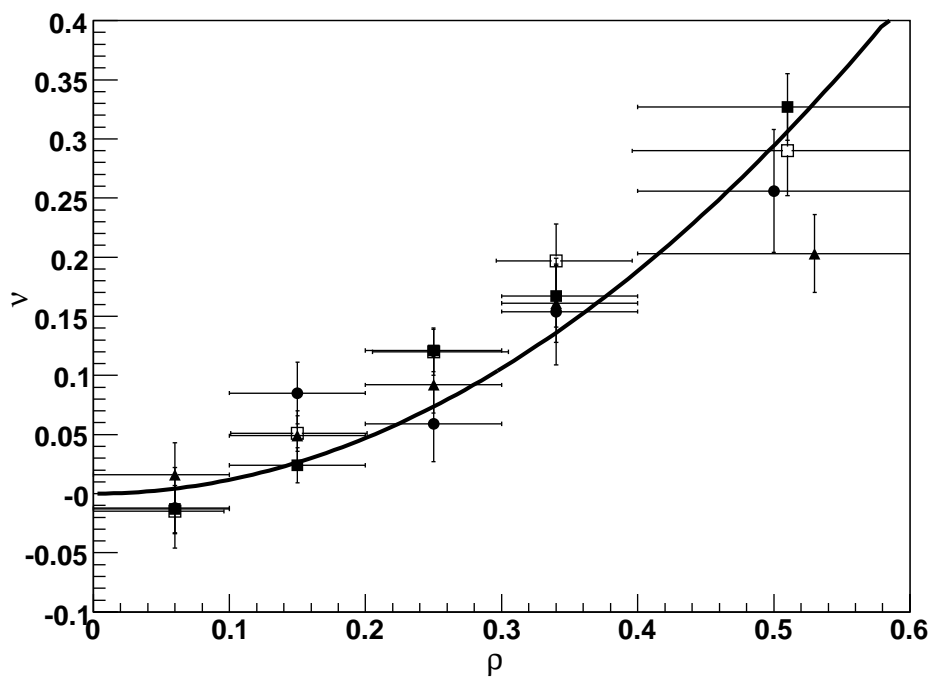

Figure 1: Azimuthal asymmetry in unpolarized DY: the asymmetry parameter $\nu$ vs $\rho=\left|\mathbf{q}_{\perp}\right| / Q$. Data are taken from the first two refs. [20]: circles correspond to $\sqrt{s}=$ 16.2 GeV, squares to $\sqrt{s}=19.1 \mathrm{GeV}$ and triangles to $\sqrt{s}=23.2 \mathrm{GeV}$. The best fit is made by means of formula (30), taking into account eq. (29). $A_{0}=1.117$.

Applying eq. (26) to SIDIS and DY yields

$$
\mu_{0}=Q / 2
$$

Moreover, results (24) to (27) can be extended, with some caution[4], to the fragmentation correlator.

It is worth comparing our approach to previous ones. Kotzinian[10] starts from the approximate expression of the density matrix for a free ultrarelativistic fermion and 
adapts it to the case of a quark in the nucleon. He parametrizes the density matrix with the 6 twist-2, T-even functions that appear in the parametrization (15). Similar results are obtained by Ralston and Soper[1] and by Tangerman and Mulders[9]. The difference with our approach is that those authors do not take into account the Politzer theorem, which implies relations among the 6 soft funtions. In any case, eq. (15) turns out to give the same result as found by Ralston and Soper[1] as regards the parametrization of the DY hadronic tensor in the limit of parton model. Indeed, according to our approximation, the symmetric hadronic tensor for two polarized protons consists of just three coefficients, corresponding, respectively, to the convolutive products $q \otimes \bar{q}, \Delta q \otimes \Delta \bar{q}$ and $\Delta_{T} q \otimes \Delta_{T} \bar{q}$.

A remark is in order. Eqs. (24), (25) and (27) have been deduced in the framework of the QCD parton model. They survive QCD evolution, but are modified by quark-gluon interactions. In particular, the Politzer theorem implies[15] that such interactions modify those approximate equalities by terms of order $g^{2} / Q^{2}$. This prediction could be tested, for example, in the case of $g_{T}$, by performing measurements at different $Q^{2}$ values.

\section{$5 \quad$ Phenomenological analysis}

Now we test the relations just found against results from three different experiments.

1) Unpolarized Drell-Yan with a fixed transverse momentum $\mathbf{q}_{\perp}$ of the final muon pair. In this case the momentum of each muon presents an azimuthal asymmetry with respect to the plane containing the initial beam direction and $\mathbf{q}_{\perp}$. This azimuthal asymmetry is essentially of the type $1 / 2 \nu \sin ^{2} \theta \cos 2 \phi[20]$, where $\theta$ and $\phi$ are respectively the polar and azimuthal angle of the $\mu^{+}$momentum in the Collins-Soper frame[21], defined in the center of mass of the pair, while $\nu$ is a dimensionless coefficient. This asymmetry is interpreted[20] in terms of quark-antiquark annihilation into a virtual photon. In the formalism of the correlator[2, 8, 12] the asymmetry is described[23] as a convolutive product of the transversities $h_{1}^{\perp}$ and $\bar{h}_{1}^{\perp}$ of the two 
active partons in the initial hadrons, providing for $\nu$ an expression of the type[12]

$$
\nu=A_{0} \frac{\mathbf{q}_{\perp}^{2}}{\mu_{0}^{2}} .
$$

But eqs. (26) and (29) imply $\mu_{0}=1 / 2 Q$, where in this case $Q$ has to be identified with the effective mass of the muon pair. Of course, also the first order perturbative QCD corrections (Compton scattering and gluon production[20]) give rise to an asymmetry parameter which, for $\left|\mathbf{q}_{\perp}\right|<<Q$, is again of the type $\mathbf{q}_{\perp}^{2} / Q^{2}$; however, these perturbative contributions fulfil the Lam-Tung relation[22], which, instead, results to be rather strongly violated[20]. Figs. 1 and 2 show comparison between formula (30) and the parameter $\nu$, as results from best fits to Drell-Yan data[12]. In this connection we observe that, owing result (29), the $Q^{2}$-dependence for DY single spin asymmetry with a tranversely polarized nucleon coincides with the one found in ref. [24].

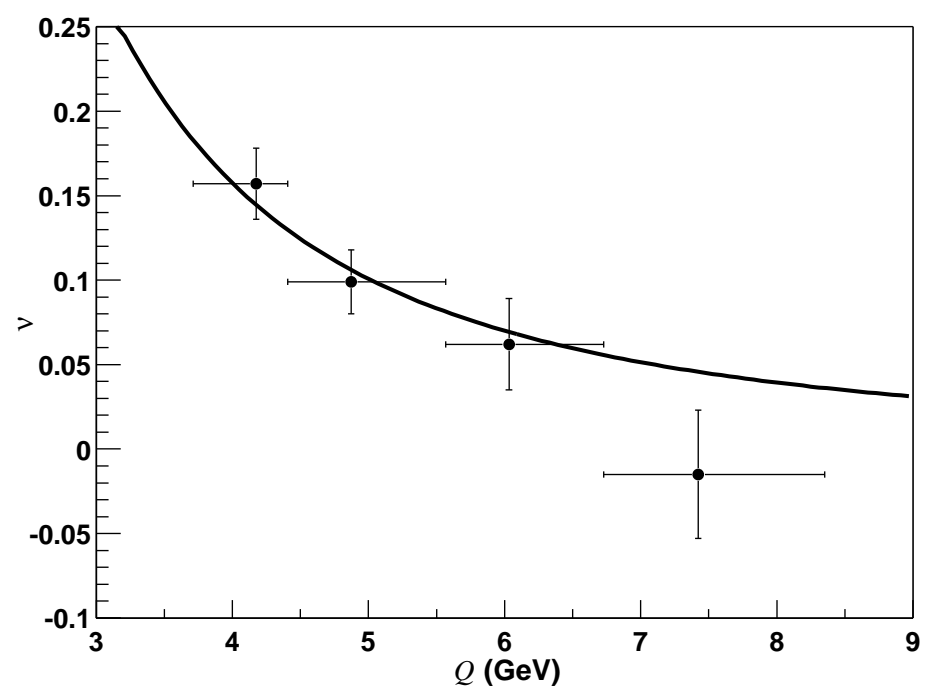

Figure 2: Azimuthal asymetry in unpolarized DY: the asymmetry parameter $\nu$ vs the effective mass $Q$ of the final lepton pair at fixed $\left|\mathbf{q}_{\perp}\right| . \sqrt{s}=23.2 \mathrm{GeV}$. Data from $2^{\text {nd }}$ ref. [20] and fitted by formula (30), taking into account eq. (29). $A_{0} \cdot \mathbf{q}_{\perp}^{2}=2.52$ $\mathrm{GeV}^{2}$.

2) CLAS[25] results of SIDIS with longitudinally polarized beam and target. The 
single spin asymmetry includes a $\sin 2 \phi$ term, characterized by the convolutive product $h_{1 L}^{\perp} \otimes H_{1}^{\perp}$, where $H_{1}^{\perp}$ is the Collins[26] fragmentation function. The function

$$
\tilde{h}_{1 L}^{\perp}(x)=\pi\left\langle\mathbf{p}_{\perp}^{2}\right\rangle h_{1 L}^{\perp}(x, \mathbf{0})
$$

has been extracted[25] for $u$-quarks from that asymmetry, taking into account a model evaluation[27] of the Collins fragmentation function and assuming a Gaussian behavior as regards the $\mathbf{p}_{\perp}^{2}$ dependence of $h_{1 L}^{\perp}\left(x, \mathbf{p}_{\perp}^{2}\right)$. However the normalization adopted in ref. [25] for the "soft" functions is the usual one[2], which, as already explained in sect. 2, is different from the one used in the parametrization (6)-(8). In particular, as regards $h_{1 L}^{\perp}$, the conversion factor is $\mathcal{C}=2 M / Q x$, according to eqs. (12) and (29): it has to be taken into account, since eqs. (25) hold within our normalization. With this re-normalization in mind, we compare (fig. 3) the CLAS[25] results of $-x h_{1 L}^{\perp u}(x)$ [from now on, the "tilde" will be dropped from eq. (31)] to the HERMES data[28] of $x g_{1}^{u}(x)$, assuming, again, a Gaussian behavior for $g_{1 L}\left(x, \mathbf{p}_{\perp}^{2}\right)$. The curve corresponds to the parametrization of $x g_{1}^{u}(x)$ given by GRSV[29] - LO, valence scenario - scaled with a factor $(1+R)^{-1}[28]$. The discrepancy, which appears for $x>0.3$, is typical of higher twist contribution, not negligible for the modest $Q^{2}$-values involved, 1.5 to 3 $(\mathrm{GeV} / \mathrm{c})^{2}$.

3) Unpolarized SIDIS $\cos \phi$ asymmetry, $\phi$ being the azimuthal angle of the final hadron momentum with respect to the reaction plane. Recently Anselmino et al.[18] fitted EMC[30] and E665[31] data by means of relation (27). They parametrized $f_{1}\left(x, \mathbf{p}_{\perp}^{2}\right)$ by making the Gaussian assumption, with $\left\langle\mathbf{p}_{\perp}^{2}\right\rangle=0.25(\mathrm{GeV} / \mathrm{c})^{2}$, and taking the MRST 2001 (LO)[32] parametrization for $f_{1}(x)$. The agreement is good. It is to be noticed, however, that the values of $x$ involved are considerably smaller than those corresponding to CLAS data, whereas the $Q^{2}$-values are much greater on the average.

\section{Conclusion and outlook}

To conclude, relations (24) to (27) have some predictive power, supported by present

data. This is a goad to further measurements and investigations in forthcoming SIDIS 


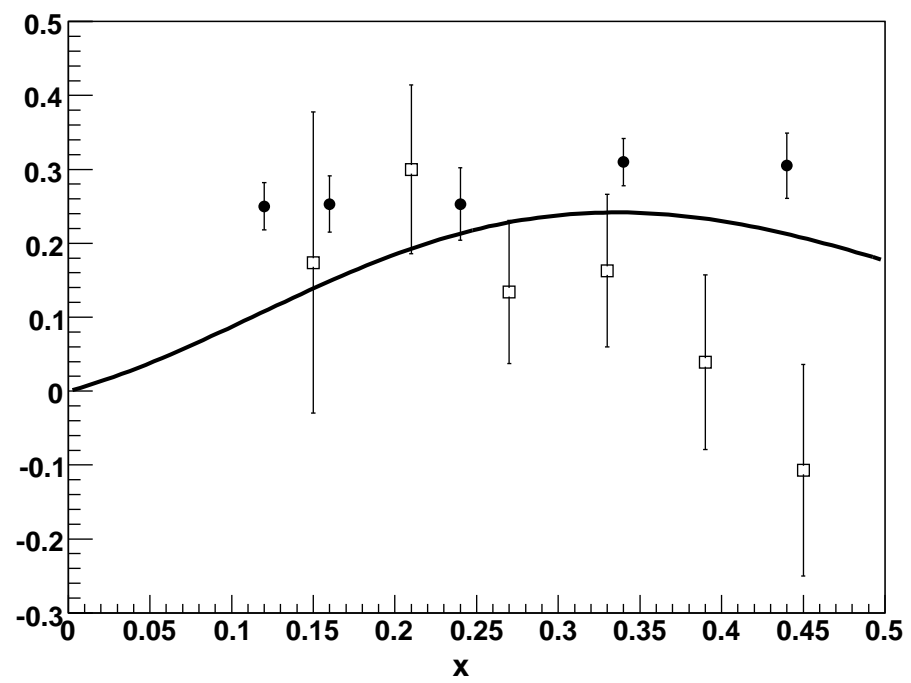

Figure 3: The functions $x g_{1}^{u}(x)$ [28] (full circles, $Q^{2}=2.5 \mathrm{GeV}^{2}$ ) and $-x h_{1 L}^{\perp u}[25]$ (open squares, $Q^{2}=1.5-3 \mathrm{GeV}^{2}$ ). The latter has been re-normalized according to the conversion factor $\mathcal{C}=2 M / Q$. The curve corresponds to the GRSV2000 parametrization (LO, valence scenario)[29] of $x g_{1}^{u}(x)$, scaled with a factor $(1+R)^{-1}[28]$.

and DY experiments.

- First of all, we suggest to determine the $\sin 2 \phi$ SIDIS azimuthal asymmetry with a longitudinally polarized target, at higher energies $(12 \mathrm{GeV})$ and at a higher precision than in ref.[25]. In particular, new extractions of $h_{1 L}^{\perp}(x)$ should take into account the recent determination of the Collins function by BELLE[33] collaboration. According to our result, this asymmetry is predicted to decrease like $Q^{-2}$. Moreover we could test our prediction about higher twist effects, according to which the sum $h_{1 L}^{\perp}(x)+g_{1}(x)$ decreases again like $Q^{-2}$. The function $h_{1 L}^{\perp}(x)$ could be determined also by means of the DY single spin asymmetry, which includes a term of the type $h_{1 L}^{\perp} \otimes \bar{h}_{1}^{\perp}$; in this case it would be suitable to realize $p \bar{p}$ collisions[34, 35] or $\pi^{-} p$ scattering[36], since the function $\bar{h}_{1}^{\perp}$ can be extracted from unpolarized DY.

- The above determinations, while interesting in themselves, would increase reliability of the approximate relations deduced above, in particular, of the last two eqs. 
(25), not directly testable at the moment, which can be exploited for determining approximately $h_{1 T}$ and $h_{1 T}^{\perp}$ through the chiral-even function $g_{1 T}$. Indeed, this may be extracted from double spin asymmetry in SIDIS, with a longitudinally polarized beam and a transversely polarized target[37]. No particular problems should arise in inferring $g_{1 T}$ from asymmetry data, since this asymmetry is characterized by the convolutive product $g_{1 T} \otimes D$, where $D$ is the common unpolarized fragmentation function of the pion.

\section{References}

[1] J. Ralston and D.E. Soper: Nucl. Phys. B 152, (1979) 109

[2] P.J. Mulders and R.D. Tangerman: Nucl. Phys. B 461 (1996) 197

[3] R.L. Jaffe and X. Ji: Phys. Rev. Lett. 67 (1991) 552; Nucl. Phys. B 375 (1992) 527

[4] A. Bacchetta, P.J. Mulders and F. Pijlman: Phys. Lett. B 595, (2004) 309

[5] K. Goeke, A. Metz, P.V. Pobylitsa and M.V. Polyakov: Phys. Lett. B 567 (2003) 27 ;

K. Goeke, A. Metz and M. Schlegel: Phys. Lett. B 618, (2005) 90

[6] A. Bacchetta, M. Boglione, A. Henneman and P.J. Mulders: Phys. Rev. Lett. 85 (2000) 712

[7] D. Boer and P.J. Mulders: Phys. Rev. D 57 (1998) 5780

[8] D. Boer, R. Jakob and P.J. Mulders: Nucl. Phys. B 564, 471 (2000)

[9] R.D. Tangerman and P.J. Mulders: Phys. Rev. D 51 (1995) 3357; Phys. Lett. B $352(1995) 129$

[10] A.M. Kotzinian: Nucl. Phys. B 441 (1995) 234

[11] M. Anselmino, A.V. Efremov and E. Leader: Phys. Rep. 261 (1995) 1 
[12] E. Di Salvo: hep-ph/0603005

[13] J. Qiu: Phys. Rev. D 42 (1990) 30

[14] H.D. Politzer: Nucl. Phys. B 172 (1980) 349

[15] E. Di Salvo: hep-ph/0511318, Talk given at "Transversity 2005", Villa Olmo, Como, 7-10 Sept. 2005

[16] S.J. Brodsky, D.S. Hwang and I. Schmidt: Phys Lett. B 530 (2002) 99; Nucl. Phys. B 642 (2002) 344; Int. Jou. Mod. Phys. A 18 (2003) 1327

[17] R.N. Cahn: Phys. Lett. B 78 (1978) 269; Phys. Rev. D 40 (1989) 3107

[18] M. Anselmino, M. Boglione, U. D'Alesio, A.M. Kotzinian, F. Murgia and A. Prokudin: Phys. Rev. D 71 (2005) 074006

[19] J.L. Cortes, B. Pire and J.P. Ralston: Z. Phys. C - Particles and Fields 55 (1992) 409

[20] NA10 Coll., S. Falciano et al.: Z. Phys. C - Particles and Fields 31 (1986) 513;

NA10 Coll., M. Guanziroli et al.: Z. Phys. C - Particles and Fields 37 (1988) 545 ;

J.S. Conway et al.: Phys. Rev. D 39 (1989) 92

[21] J.C. Collins and D.E. Soper: Phys. Rev. D 16 (1977) 2219

[22] C.S. Lam and W.K. Tung: Phys. Rev. D 21 (1980) 2712

[23] D. Boer, S. Brodsky and D.-S. Huang: Phys. Rev. D 67 (2003) 054003

[24] D.Boer, P.J. Mulders O.V. Teryaev: Phys. Rev. D 57 (1998) 3057

[25] H. Avakian, P. Bosted, V. Burkert and L. Elouadrhiri: AIP Conf. proc. 792 (2005) 945

[26] J.C. Collins: Nucl. Phys. B 396 (1993) 161 
[27] A.V. Efremov et al.: Phys. Rev. D 77 (2003) 114014

[28] HERMES Coll., A. Airapetian et al.: Rev. D 71 (2005) 012003

[29] M. Glueck, E. Reya, M. Stratmann and W. Vogelsang: Phys. Rev. D 63 (2001) 094005

[30] EMC Coll., M. Arneodo et al.: Z. Phys. C 34 (1987) 277; J. Ashman et al.: Z. Phys. C 52 (1991) 361

[31] E665 Coll., M.R. Adams et al.: Phys. Rev. D 48 (1993) 5057

[32] A.D. Martin, R.G. Roberts, W.J. Stirling and R.S. Thorne: Phys. Lett. B 531 (2002) 216

[33] BELLE Coll., K. Abe et al.: hep-ex/0705063

[34] PAX Coll., "Antiproton-Proton Scattering Experiments with Polarization", Julich, April 2005;

M. Kotulla et al.: Technical Progress Report for PANDA (2005), http://www.ep1.rub.de/ ${ }^{\sim}$ panda/db/papersdb/pc19-050217_ panda.tpr.pdf

[35] A. Sissakian et al.: Phys. Rev. D 72 (2005) 054002

[36] A. Sissakian et al.: Eur. Phys. J. C 46 (2006) 147

[37] A.M. Kotzinian and P.J. Mulders: Phys. Rev. D 54 (1996) 1229 